\section{Hépatite \\ chronique C \\ à transaminases \\ normales de façon \\ répétée}

Christophe Hezode, Daniel Dhumeaux

> Environ un quart des malades atteints d'hépatite chronique $C$ ont des transaminases normales de façon répétée. Le pronostic est dans l'ensemble bon. La vitesse de progression de la fibrose est lente et l'évolution vers la cirrhose est rare dans cette population. En raison d'une faible efficacité des traitements disponibles, l'attitude thérapeutique recommandée était jusqu'à présent l'abstention. Avec l'arrivée de la bithérapie interféron pégylé-ribavirine, beaucoup plus efficace, l'opportunité de traiter ces malades doit être rediscutée, en particulier chez ceux qui ont une forte probabilité de réponse prolongée. <

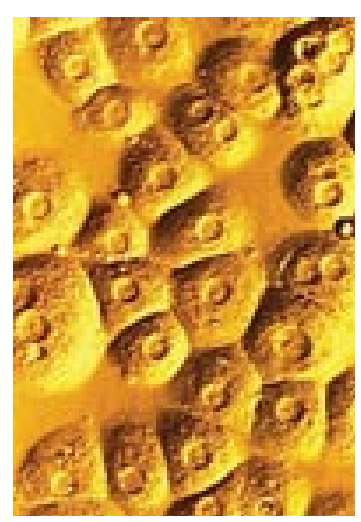

de la période d'observation varient selon les études, cette entité « hépatite $C$ à transaminases normales » reste difficile à définir car les normes des ALAT fournies par les laboratoires d'analyse ont généralement été établies à partir d'un nombre limité de sujets, et ne tiennent compte ni du sexe, ni de l'index de masse corporelle de ces sujets. Or, le seuil de normalité des ALAT dépend de ces paramètres [1].

La prévalence des malades ayant une hépatite chronique $C$ à transaminases normales de façon répétée est mal connue. En 2000, il a été rapporté que 0,08\% des donneurs de sang en France ont des anticorps anti-VHC [2]. Parmi ces donneurs de sang porteurs d'anticorps anti-VHC, $27 \%$ à $59 \%$ ont des transaminases normales et chez $54 \%$ à $65 \%$ d'entre eux la recherche de l'ARN viral est positive dans le sérum [3-5]. A partir de ces données, la proportion de malades ayant une hépatite chronique $C$ à transaminases normales serait de $25 \%$. Dans des centres cliniques qui ont déterminé la prévalence de ces malades au sein de leur série personnelle, celle-ci variait de 5\% à 39\% [6-10]. Chez nos propres malades, elle est de $14 \%$. 


\section{Qui sont ces malades?}

Les modes de contamination par le VHC, comme la transfusion sanguine ou l'usage de drogue intraveineuse, sont observés avec la même fréquence chez les malades ayant des transaminases normales ou élevées $[3,5,7,8,11,12]$. Les études qui ont comparé les caractéristiques cliniques des malades ayant des transaminases normales ou augmentées n'ont pas noté de différences significatives. Cependant, un âge moyen plus élevé et une proportion plus importante de femmes ont parfois été rapportés chez les premiers $[3,5,11]$. La prépondérance féminine pourrait être liée à une maladie plus bénigne chez la femme, mais aussi au seuil définissant la normalité des transaminases: dans la population des donneurs de sang, la limite supérieure de la normale des ALAT était plus élevée chez l'homme que chez la femme [1], une caractéristique qui n'est pas prise en compte pour établir les normes des laboratoires. Dans le même ordre d'idée, il serait intéressant de comparer l'index de masse corporelle chez les malades ayant des transaminases normales et ceux dont les ALAT sont élevées.

L'étude de la distribution des génotypes des hépatites chroniques $C$ avec transaminases normales montre que le génotype $1 b$ est le plus fréquent, suivi du génotype $3 a$, à l'exception d'une étude montrant une prépondérance du génotype 2 [9]. Lorsque cette distribution est comparée à celle observée chez des malades à transaminases élevées, aucune différence n'est constatée (Tableau I) [13, 14]. Les charges virales mesurées chez ces patients varient selon les études, mais se révèlent le plus souvent similaires $[4,6,9,11]$, parfois inférieures [8] aux autres patients. Ce résultat s'accorde avec l'hypothèse, généralement admise, que le VHC n'est pas directement cytopathogène. La variabilité génétique du VHC, étudiée à deux reprises, semble identique, que les transaminases soient normales ou augmentées [12, 15].

Sur le plan histologique, la majorité des malades ont un foie normal ou une activité histologique minime $[7,14,16]$ et la prévalence de la cirrhose est extrêmement faible dans cette population, variant de $0 \%$ à $9 \%[7,8,10,11,14,16]$ (Tableau II). Une seule étude indique une proportion importante $(20 \%)$ de fibrose sévère ou de cirrhose, mais il faut souligner que, dans cette étude, le nombre de patients est faible et certains n'ont pas des transaminases strictement normales, les valeurs atteignant 1,4 fois la limite supérieure de la normale [17]. L'étude comparée des lésions histologiques montre un score d'activité histologique (inflammation portale, lobulaire et nécrose périportale), un score de fibrose et une prévalence de la cirrhose significativement plus faibles chez les malades ayant des transaminases normales $[7,8,10,11]$. Si la conférence de consensus internationale de 1999 a recommandé de ne pas réaliser de biopsie hépatique chez ces malades [18], les progrès thérapeutiques actuels, comme on le verra plus loin, pourraient modifier cette attitude car l'intensité des lésions histologiques pourrait intervenir dans la décision de traitement ou d'abstention.

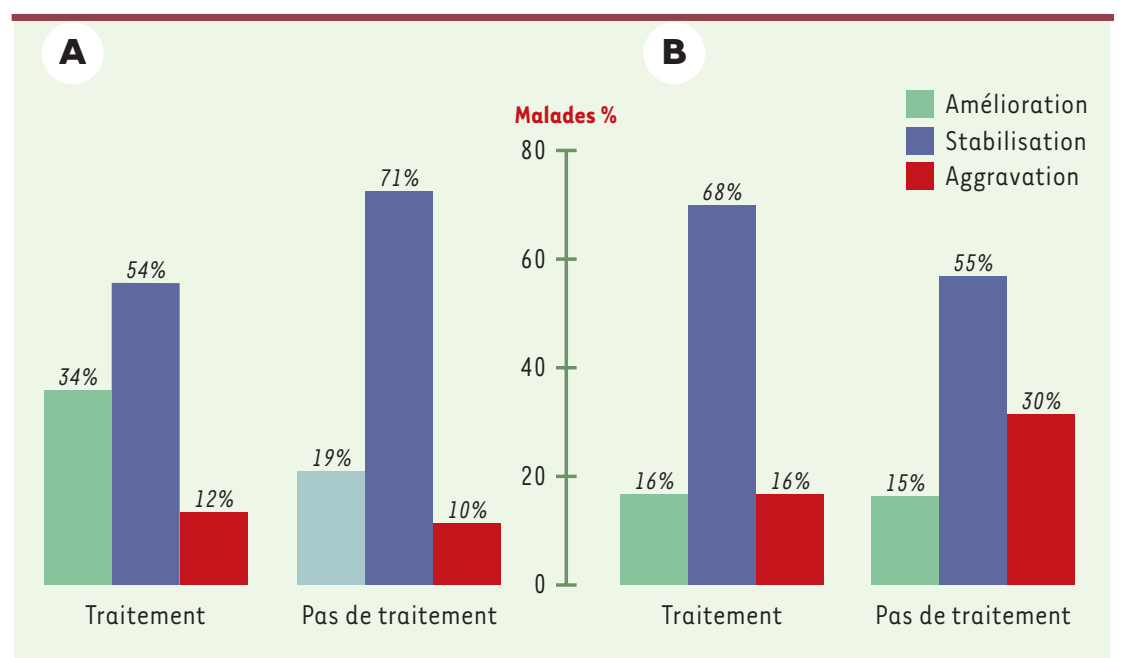

Figure 1. Étude comparée de l'évolution histologique chez des malades ayant une hépatite chronique C à transaminases normales de façon répétée, traités par interféron ou non traités [29]. A. Évolution de l'activité histologique. B. Évolution de la fibrose histologique.

\begin{tabular}{|c|c|c|c|c|}
\hline \multirow[t]{2}{*}{ Génotype } & \multicolumn{2}{|c|}{ Marcellin et al. [13] } & \multicolumn{2}{|c|}{ Tassopoulos et al. [14] } \\
\hline & $\begin{array}{l}\text { ALAT normales } \\
\quad(n=54)\end{array}$ & $\begin{array}{l}\text { ALAT élevées } \\
\quad(n=58)\end{array}$ & $\begin{array}{l}\text { ALAT normales } \\
\quad(n=81)\end{array}$ & $\begin{array}{l}\text { ALAT élevées } \\
(n=171)\end{array}$ \\
\hline 1 * & $3 \%$ & $3 \%$ & $5 \%$ & $5 \%$ \\
\hline la & $22 \%$ & $19 \%$ & $5 \%$ & $6 \%$ \\
\hline lb & $50 \%$ & $28 \%$ & $38 \%$ & $35 \%$ \\
\hline 2 & $10 \%$ & $12 \%$ & $14 \%$ & $7 \%$ \\
\hline 3 & $6 \%$ & $35 \%$ & $19 \%$ & $35 \%$ \\
\hline Autres & $9 \%$ & $3 \%$ & $20 \%$ & $13 \%$ \\
\hline * non sous- & typé avec le test & tilisé & & \\
\hline
\end{tabular}

Tableau I. Comparaison dans deux séries $[13,14]$ de la prévalence des génotypes du VHC chez des malades ayant des transaminases normales de façon répétée ou des transaminases élevées. 
Quelle est le risque évolutif ?

Le pronostic des hépatites chroniques C à transaminases normales de façon répétée est bon. La plupart des

\begin{tabular}{lccc}
\hline Études & $\begin{array}{c}\text { Foie normal } \\
\text { ou activité minime }\end{array}$ & Activité modérée & Cirrhose \\
Marcellin et al. [16] $(n=293)$ & $81 \%$ & $19 \%$ & $1 \%$ \\
Tassopoulos et al. [13] $(n=169)$ & $90 \%$ & $10 \%$ & $0,3 \%$ \\
Mathurin et al. [7] $(n=66)$ & $94 \%$ & $6 \%$ & $1 \%$ \\
Jamal et al. [8] $(n=75)$ & - & - & $6 \%$ \\
Healey et al. [10] $(n=23)$ & - & - & $9 \%$ \\
Shiffman et al. [14] $(n=37)$ & - & - & $6 \%$ \\
Nutt et al. $[17](n=35)$ & - & - & $11 \%$ \\
\hline
\end{tabular}

Tableau II. Analyse des principales études histologiques publiées chez les malades ayant une hépatite chronique $\mathrm{C}$ à transaminases normales de façon répétée.

\begin{tabular}{|c|c|c|c|}
\hline $\begin{array}{l}\text { Études } \\
\text { Études randomisées }\end{array}$ & $\begin{array}{l}\text { Nombre de } \\
\text { malades }\end{array}$ & Type de traitement & $\begin{array}{c}\text { Réponse } \\
\text { virologique } \\
\text { durable (\%) }\end{array}$ \\
\hline \multirow[t]{2}{*}{ Sangiovanni et al. [24] } & 16 & $3 \mathrm{MU} \times 3 /$ semaine 6 mois & 0 \\
\hline & 15 & Pas de traitement & 0 \\
\hline \multirow[t]{2}{*}{ Vafiades et al. [14] } & 37 & $5 \mathrm{MU} \times 3 /$ semaine 6 mois & 22 \\
\hline & 39 & Pas de traitement & 5 \\
\hline \multirow[t]{2}{*}{ Rossini et al. [15] } & 10 & $3 \mathrm{MU} \times 3 /$ semaine 12 mois & 20 \\
\hline & 9 & Pas de traitement & 0 \\
\hline \multirow[t]{2}{*}{ Hézode et al. [26] } & 27 & $3 \mathrm{MU} \times 3 /$ semaine 12 mois & 15 \\
\hline & 23 & Pas de traitement & 0 \\
\hline \multirow[t]{2}{*}{ Tran et al. [27] } & 11 & $3 \mathrm{MU} \times 3$ /semaine 12 mois & 18 \\
\hline & 20 & Pas de traitement & 0 \\
\hline \multirow[t]{2}{*}{ Shiffman et al. [10] } & 35 & $\begin{array}{l}3 \text { MU/jour } 3 \text { mois } \\
\text { puis } 3 \text { MUx } 3 / \text { semaine } 12 \text { mois }\end{array}$ & 11 \\
\hline & & $3 \mathrm{MU} \times 3 /$ semaine 18 mois & 8 \\
\hline \multicolumn{4}{|l|}{ Études ouvertes } \\
\hline Serfaty et al. [19] & 10 & 3 MUx3/semaine 6 mois & 0 \\
\hline Areias et al. [20] & 14 & $3 \mathrm{MU} \times 3 /$ semaine 6 mois & 21 \\
\hline Ideo et al. [21] & 16 & $3 \mathrm{MU} \times 3 /$ semaine 6 mois & 13 \\
\hline Silverman et al. [22] & 15 & $3 \mathrm{MU} \times 3 /$ semaine 6 mois & 0 \\
\hline Nordoy et al. [23] & 23 & $3 \mathrm{MU} \times 3 /$ semaine 6 mois & 9 \\
\hline Orito et al. [25] & 23 & $10 \mathrm{MU} \times 3 /$ semaine 6 mois & 37 \\
\hline Van Thiel et al. [28]* & 21 & $5 \mathrm{MU} /$ jour 12 mois & non rapportée \\
\hline \multirow[t]{2}{*}{ Lee et al. [30] } & 19 & $\begin{array}{l}5 \mathrm{MU} / \text { jour } 1 \text { mois puis } \\
3 \mathrm{MU} 3 / \text { semaine } 11 \text { mois }\end{array}$ & 47 \\
\hline & & $\begin{array}{l}\text { + ribavirine } \\
\text { ( } 1 \text { à } 1,2 \mathrm{~g} / \text { jour en fonction du poids) }\end{array}$ & 47 \\
\hline
\end{tabular}

Tableau III. Efficacité du traitement antiviral en terme de réponse virologique durable chez des malades ayant des transaminases normales de façon répétée. patients ont déjà, au moment du diagnostic, une longue évolution de leur infection (jusqu'à 20 ou 30 ans, sans lésion histologique sévère). Persico et al. [9] ont étudié de façon prospective l'histoire naturelle de ces hépatites par des biopsies répétées à 5 ans d'intervalle chez 24 malades dont les transaminases étaient strictement normales sur une période de 5 ans. Aucune aggravation significative de l'activité histologique et de la fibrose n'a été observée quel que soit le génotype du VHC [9]. La vitesse moyenne annuelle de progression de la fibrose est nettement plus lente que celle observée chez les patients ayant des transaminases élevées [7], un score de fibrose important n'étant observé que chez les sujets ayant des transaminases normales mais consommant plus de $50 \mathrm{~g}$ d'alcool par jour [7].

\section{Quel est le traitement?}

Sept études ouvertes et six essais randomisés ont évalué l'efficacité de l'interféron standard en monothérapie chez ces malades (Tableau III) [10, 14, 15, 1928]. Aucun malade n'avait été traité auparavant et les schémas thérapeutiques utilisés étaient multiples. Les doses d'interféron prescrites étaient de 3 à 10 millions d'unités internationales trois fois par semaine ou 5 millions d'unités internationales par jour, et la durée d'administration s'étendait de 6 à 18 mois. Globalement, le taux de réponse virologique durable (ARN viral négatif six mois après l'arrêt du traitement) était de l'ordre de $15 \%$ (Tableau III) [10, 14, 15, 19-28]. Ce résultat est identique à celui observé chez les malades ayant des transaminases élevées [10]. Si une augmentation des transaminases a été observée pendant le traitement et dans les six mois qui ont suivi son arrêt, chez $47 \%$ des patients $[14,26]$, cette augmentation, à l'exception d'une étude [24], ne semble pas différente de celle observée chez les malades non traités [14, 26]. Dans notre étude, l'évolution histologique (score METAVIR) n'a pas été significativement différente chez les malades traités par interféron ou non traités [29]. Toutefois, une amélioration 
de l'activité histologique était observée respectivement dans $34 \%$ versus $19 \%$ des cas et une aggravation de la fibrose dans $16 \%$ versus $30 \%$ des cas (Figure 1 ).

Seules deux études ont évalué l'efficacité d'un traitement associant l'interféron et la ribavirine dans l'hépatite chronique $C$ à transaminases normales ou proches de la normale. Dans la première, une étude ouverte portant sur 19 malades naïfs [30], le traitement était poursuivi pour 36 semaines supplémentaires si le virus était indétectable dans le sérum à la douzième semaine et interrompu dans le cas contraire. Une réponse virologique durable a été observée chez la moitié des malades, et il n'a pas été constaté d'augmentation des transaminases durant le traitement. Dans la seconde, une réponse virologique durable a été rapportée chez $30 \%$ des malades ayant des transaminases $<1,3$ fois la normale, un taux voisin de celui obtenu quand les transaminases étaient plus élevées (37\%) [31].

Enfin, en ce qui concerne l'association interféron pégyléribavirine dont la supériorité thérapeutique sur la bithérapie standard a été clairement démontrée [32, 33], puisqu'on obtient jusqu'à 76 et $79 \%$ de réponse virologique prolongée pour un virus de génotype 2 ou 3 , aucune donnée concernant son efficacité n'est disponible à l'heure actuelle chez les malades ayant des transaminases normales. Par analogie avec l'interféron en monothérapie et la bithérapie utilisant l'interféron standard, il est probable que les résultats seront similaires.

La conférence de consensus internationale de 1999 avait recommandé de ne pas traiter ces malades en raison des lésions histologiques souvent minimes et de l'efficacité insuffisante de l'interféron en monothérapie [18]. Si les résultats encourageants de la bithérapie pégylée sont confirmés par les études en cours, un traitement pourrait être proposé aux malades ayant des transaminases normales et ayant notamment une forte probabilité de réponse virologique.

\section{Conclusions}

Environ un quart des malades infectés par le VHC ont une hépatite chronique associée à des transaminases normales de façon répétée. Chez ces patients, l'atteinte histologique est le plus souvent minime et la présence d'une cirrhose est rare. Jusqu'à présent, l'attitude thérapeutique était l'abstention, justifiée par l'efficacité réduite des traitements et le risque évolutif généralement faible. Avec les traitements dont on dispose actuellement, beaucoup plus efficaces, l'opportunité de traiter ces malades doit être à nouveau discutée. La décision d'instaurer un traitement pourrait reposer sur les paramètres suivants: l'âge du malade, le génotype

du VHC et la sévérité de l'atteinte histologique. Un traitement pourrait être proposé d'autant plus facilement que le sujet est jeune, a un génotype favorable et une activité et/ou une fibrose notable à la biopsie hépatique. II nous faut attendre sur ce sujet les recommandations de la conférence de consensus française de février 2002. $\diamond$

\section{SUMMARY}

Chronic hepatitis $C$ with repeatedly normal serum transaminases

An estimated $25 \%$ of patients with chronic hepatitis C virus (HCV) infection have persistently normal serum alanine aminotransferase (ALAT). These patients have only mild hepatitis and they may either not progress to cirrhosis at all or progress at a much slower rate than persons with elevated serum ALT levels. Given the limited response to IFN and IFN-ribavirin combination therapy, it was recommended that these patients should not be usually treated. Because of the great improved response rate observed with pegIFN plus ribavirine, these recommendations will probably be discussed again in patients with a high probability of sustained response. $\diamond$

\section{RÉFÉRENCES}

1. Piton A, Poynard T, ImbertBismut $F$, et al. Factors associated with serum alanine transaminase activity in healthy subjects: consequences for the definition of normal values, for selection of blood donors, and for patients with chronic hepatitis C. Hepatology $1998 ; 27: 1217-9$.

2. Pillonel J, Laperche $S$. Surveillance des marqueurs d'une infection par le VIH, I'HTLV et les virus des hépatites $B$ et $C$ chez les donneurs de sang en France de 1991 à 2000. Bull Epidemiol Hebdo 2001 ; 46 : 207-9.

3. McGuinness PH, Bishop GA, Lien A, Wiley B, Parsons C, McCaughan. Detection of serum hepatitis C virus RNA
Fleming KA. Liver histology in hepatitis $C$ infection: a comparison between patients with persistently normal or abnormal transaminases. Gut 1995 ; 37 : 274-8.

4. Prieto M, Olaso V, Verdu C, et al. Does the healthy hepatitis C virus carrier state really exist? An analysis using polymerase chain reaction. Hepatology 1995 ; $22: 413-7$.

5. Shakil AO, Conry-Cantilena C, Alter HJ, et al. Volunteer blood donors with antibody to hepatitis $C$ virus: clinical, biochemical, virologic, and histologic features. Ann Intern Med 1995 ; 123 : 330-7.

6. Gholson CF, Morgan K, Catinis G, et al. Chronic hepatitis $C$ with normal 
aminotransferase levels. A clinical histologic study. Am J Gastroenterol 1997 ; 92 : 1788-92.

7. Mathurin P, Moussalli J, Cadranel JF, et al. Slow progression rate of fibrosis in hepatitis $C$ virus patients with persistently normal alanine transaminase activity. Hepatology 1998 ; 27 : 868-72.

8. Jamal MM, Soni A, Quinn PG, Wheeler DE, Arora S, Johnston DE. Clinical features of hepatitis $\mathrm{C}$ infected patients with persistently normal alanine transaminase levels in the Southwestern United States. Hepatology 1999 ; 30 : 1307-11.

9. Persico M, Persico $\varepsilon$, Suozzo $R$, et al. Natural history of hepatitis $C$ virus carriers with persistently normal aminotransferase levels. Gastroenterology 2000 ; 118 : 760-4.

10. Shiffman ML, Stewart CA, Hofmann CM, et al. Chronic infection with hepatitis C virus in patients with elevated or persistently normal serum alanine aminotransferase levels: comparison of hepatic histology an response to interferon therapy. J Infect Dis 2000 ; 182 : 1595-601.

11. Healey CJ, Chapman RW, Fleming KA. Liver histology in hepatitis C infection: a comparison between patients with persistently normal or abnormal transaminases. Gut 1995 ; 37: 274-8.

12. Shindo M, Arai K, Sokawa $Y$, Okuno T. The virological and histological states of antihepatitis C virus-positive subjects with normal liver biochemical values. Hepatology 1995 ; 22 : 418-25.

13. Marcellin P, Kilani A, Cymes $K$, et al. Virological and histological characteristics in anti-HCV positive subjects with normal transaminase levels (abstract). Hepatology 1995 ; 22 : 273A.

14. Tassopoulos NC. Treatment of patients with chronic hepatitis C and normal ALT levels. J Hepatol 1999 ; 31 (suppl 1) : 193-6.

15. Rossini A, Ravaggi A, Biasi $L$, et al. Virological response to interferon treatment in hepatitis $C$ virus carriers with normal aminotransferase levels and chronic hepatitis. Hepatology 1997 ; 26 : 1012-7.

16. Marcellin P, Lévy $S$, Erlinger S. Therapy of hepatitis $C$ : patients with normal aminotransferase levels. Hepatology 1997; 26 (suppl 1) : 133S-136S.

17. Nutt AK, Hassan HA, Lindsey J, Lamps LW, Raufman JP. Liver biopsy in the evaluation of patients with chronic hepatitis $C$ who have repeatedly normal or nearnormal serum alanine aminotransferase levels. Am J Med $2000 ; 109: 62-4$.

18. EASL International Consensus Conference on Hepatitis C. Consensus statement. J Hepatol 1999 ; 30 : 956-61.

19. Serfaty $L$, Chazouillères 0 , Pawlotsky JM, Andreani T, Pellet C, Poupon R. Interferon alpha therapy in patients with chronic hepatitis $C$ and persistently normal aminotransferase activity. Gastroenterology 1996 ; 110 : 291-5.

20. Areias J, Pedroto I, Freitas $\mathrm{T}$, et al. Hepatitis C virus carriers with normal ALT activity: viremia, genotype and effect of interferon therapy (abstract). Gastroenterology 1996 ; 110 : 1144A.

21. Ideo G, Bellobuono A, Temprini S, Mondazzi L, Bellati G, Zanetti AR. Poor efficacy of alpha-interferon treatment in patients affected by chronic hepatitis $C$ with normal or near normal ALT levels (abstract). Gastroenterology 1996 ; 110 : 1215A.

22. Silverman $A L$, Piquette $D L$, Filipiak CL, Neill JS, Bayati $\mathrm{N}$, Gordon SC. Alfa-interferon treatment of hepatitis $C$ virus RNA-positive patients with normal or near-normal alanine aminotransferase levels. Am J Gastroenterol 1997 ; 92 : 1793-5.

23. Nordoy I, Krarup HB, Bell H, et al. Interferon-alpha $2 \mathrm{~b}$ therapy in low-activity hepatitis C: a pilot study. Scand J Gastroenterol 1997 ; 32 : 1256-60.

24. Sangiovanni A, Morales $R$, Spinzi GC, et al. Interferon alfa treatment of HCV RNA carriers with persistently normal transaminase levels: a pilot randomized controlled study. Hepatology $1998 ; 27: 853-6$.

25. Orito $\varepsilon$, Mizokami M, Suzuki $K$, et al. Interferon alpha therapy for individuals with normal serum ALT before treatment. J Gastroenterol Hepatol 1996 ; 12 : 58-61.

26. Hézode C, Roudot-Thoraval F, Capron D, et al. Alpha 2A interferon monotherapy in patient with chronic hepatitis $C$ and repeatedly normal aminotransferase levels (abstract). Hepatology 2000 ; $32: 352 \mathrm{~A}$

27. Tran A, Longo F, Ouzan D, et al. Effects of 1 -year Interferon-alpha 2a treatment for patients with chronic hepatitis $C$ and persistently normal transaminase activity. Scand J Gastroenterol 2000 ; 4 : 433-7.

28. Van Thiel DH, Caraceni P, Molloy PJ, et al. Chronic hepatitis $C$ in patients with normal or near normal alanine aminotransferase levels: the role of interferon alpha $2 b$ therapy. J Hepatol 1995 ; 23 : 503-8.

29. Hézode C, Roudot-Thoraval F, Capron D, et al. Histological evaluation of alpha 2 a interferon monotherapy in patients with chronic hepatitis $C$ and repeatedly normal aminotransferase levels. $\mathrm{A}$ randomized study (abstract). J Hepatol 2001 ; 34 (suppl 1) : 4A.

30. Lee SS, Sherman M. Pilot study of interferon- $a$ and ribavirin treatment in patients with chronic hepatitis $C$ and normal transaminase values. J Viral Hep 2001 ; 8 : 202-5.
31. Gordon SC, Fang JWS, Silverman AL, MCHutchison JG, Albrecht JK. The significance of baseline serum alanine aminotransferase on pretreatment disease characteristics and response to antiviral therapy in chronic hepatitis $C$. Hepatology 2000 ; 32 : 400-4.

32. Dhumeaux D, Hézode C, Fried MW, et al. Effets de l'interféron alpha 2 a pégylé (Pégasys) associé à la ribavirine dans l'hépatite chronique virale $C$. Étude randomisée multicentique (abstract). Gastroenterol Clin Biol 2001 ; 25 : 617A.

33. Manns MP, McHutchison JG, Gordon SC, Rustgi VK, Shiffman M, Reindollar $R$, et al. Peginterferon alfa-2b plus ribavirin compared with interferon alfa-2b plus ribavirin for initial treatment of chronic hepatitis $C$ : a randomized trial. Lancet 2001 ; 358 : 958-65.
TIRÉS À PART

C. Hezode 